\title{
Lactobacillus rhamnosus GG treatment improves intestinal permeability and modulates microbiota dysbiosis in an experimental model of sepsis
}

\author{
LUFANG CHEN, HANYU LI, JINYOU LI, YUE CHEN and YUNMEI YANG \\ Department of Geriatrics, First Affiliated Hospital, School of Medicine, Zhejiang University, \\ Hangzhou, Zhejiang 310003, P.R. China
}

Received August 21, 2017; Accepted May 3, 2018

DOI: $10.3892 / \mathrm{ijmm} .2019 .4050$

\begin{abstract}
Decrease of 'health-benefiting' microbes and increase of pathogenic bacteria (a condition termed dysbiosis) in intensive care unit patients is considered to induce or aggravate sepsis (gut-origin sepsis). Orally administered probiotics have been effective in the prevention of nosocomial infections. However, the mechanisms of probiotic-induced anti-infection and anti-sepsis remain to be explored. In the present study, 4-week-old C57BL6 mice were orally administrated with Lactobacillus rhamnosus GG (LGG) or normal saline (control) 4 weeks prior to cecal ligation and puncture (CLP). A subset of the mice were sacrificed at $24 \mathrm{~h}$ post-CLP, and the others were used for survival studies. Ileum tissues, blood and fecal samples were collected. The survival rate of septic mice pretreated with LGG was significantly improved compared with untreated mice. The levels of inflammatory cytokines were reduced in LGG-pretreated septic mice. A decrease of colonic proliferation and epithelial tight junctions and an increase of colonic apoptosis were observed in control septic CLP+saline mice. LGG pretreatment reversed the colonic proliferation, apoptosis and expression of tight junction proteins to the levels of the sham group. LGG pretreatment improved the richness and diversity of intestinal microbiota in septic mice. The principal coordinates analysis clustering plots revealed a significant separate clustering in microbiota structure between three groups. Bacteria associated with energy consumption, including Bacteroidetes, with opportunistic infection, including Proteobacteria, Staphylococcaceae and Enterococcaceae, lipopolysaccharide producers, including Enterobacteriaceae, and facultative anaerobes, such as Bacteroidaceae and Erysipelotrichaceae, increased in septic mice. By contrast, bacteria associated with
\end{abstract}

Correspondence to: Dr Yunmei Yang, Department of Geriatrics, First Affiliated Hospital, School of Medicine, Zhejiang University, 79 Qingchun Road, Hangzhou, Zhejiang 310003, P.R. China E-mail: 1194070@zju.edu.cn

Key words: Lactobacillus rhamnosus GG, cecal ligation and puncture, sepsis, 16SrRNA, microbiota energy harvest, including Firmicutes, intestinal barrier function regulators, including Akkermansia, hepatic function regulators, including Coprococcus and Oscillospira, and obligate anaerobes, including Prevotellaceae, decreased in septic mice. With LGG pretreatment, the sepsis-induced microbiota dysbiosis was reversed. The present results elucidated the potential mechanism of LGG treatment in sepsis, by improving intestinal permeability and modulating microbiota dysbiosis.

\section{Introduction}

Sepsis is one of the leading causes of mortality and morbidity in children and adults (1). An altered gut microflora is always present in patients with sepsis, which induces infective and non-infective complications (2). In addition, intestinal epithelium dysfunction and dysbiosis, induced by critical illnesses, result in increased translocation of bacteria to the blood, which contributes to the adverse outcome of sepsis (3).

Probiotics are regarded as the living microorganisms, which, in adequate amounts, can bring health benefits to the host (4). Among them, the genera Lactobacillus and Bifidobacterium are the most widely used (5). Up to date, probiotics have been increasingly applied and studied in clinical practice. It is believed that probiotics can reduce the risk of disease (including diarrhea, allergic diseases and inflammatory bowel disease) through competition for binding locus and nutrients with pathogens, producing bacteriocins to kill pathogens, synthesizing IgA to modify immune responses and reducing inflammation by stimulating regulatory lymphocytes through interleukin (IL)-10 and transforming growth factor signaling (6-8). However, application of probiotics on sepsis has been limited due to the theoretical risk of aggravating bacteremia in patients with critical illnesses, although few data exist that support this concern (9). A previous study from our group has previously reported that prophylactic administration of a special probiotic bacterial species in a septic mouse model effectively reduced mortality (10). However, the underlying mechanisms by which probiotics alleviated the severity of sepsis remains unclear. In order to get a better understanding of the role that the gut microbiota serve in the process of sepsis, the present study performed a comprehensive analysis of the microbiota alterations during sepsis. 
In the past, the colonization patterns in septic patients were investigated predominantly from culture-dependent studies. However, most of the intestinal bacteria are obligate or facultative anaerobic bacteria, which are technically challenging to culture once exposed in oxygen (11). Therefore, previous studies merely confirm the absence or presence of specific bacteria, without providing a global view of microbiota alterations during sepsis. Due to the development of bacterial $16 \mathrm{~S}$ ribosomal DNA gene sequencing (12), the present study was able to decipher microbial diversity and reveal the alterations of the gut microbiota structure during sepsis. The present study aimed to investigate the effects of probiotic LGG on the microbial diversity in septic mice, and to examine how this contributes to the prevention and reversion of sepsis.

\section{Materials and methods}

Ethics statement. All procedures for animal care and use were approved by the Animal Care Ethics Committee of the First Affiliated Hospital, Zhejiang University (Hangzhou, China). Four-week-old male C57BL6 mice were purchased from Zhejiang University and housed in pathogen-free animal facilities under a standard 12-h light/dark cycle. Standard mouse diet and water were given throughout the study.

Probiotic administration and cecal ligation and puncture (CLP) model. Four-week-old male C57BL6 mice (weight, $13.59 \pm 1.59 \mathrm{~g}$ ) were administered daily by oral gavage with $200 \mu \mathrm{l}$ of $\mathrm{LGG}\left(2 \times 10^{9} \mathrm{CFU} / \mathrm{ml}, 2.9 \times 10^{7} \mathrm{CFU} / \mathrm{g}\right.$; Culturelle, ConAgra Foods, Omaha, NE, USA; CLP+LGG group, n=23), or normal saline (control sham group, $n=20 ; C L P+$ saline group, n=18) 4 weeks prior to CLP. To establish the murine septic peritonitis model, the mice were anesthetized with isoflurane and bupivacaine: 3-4\% for induction and 1-3\% for maintenance. A $1 \mathrm{~cm}$ incision was made on the middle of abdomen, and the cecum was exteriorized through the incision carefully. In order to induce mid-grade sepsis, the cecum was ligated at middle of the bottom and distal pole of the cecum, and was punctured from mesenteric toward anti-mesenteric direction using a 23-gauge needle. A droplet of feces was extruded through the holes, and the cecum was relocated into the abdominal cavity. Finally, the fascia and skin incision were closed in layers. Sham mice underwent the same operation except from the cecum ligation and puncture procedures. In all mice, $1 \mathrm{ml}$ pre-warmed normal saline was injected subcutaneously following the CLP operation (13).

Serum sample preparation. Blood samples were obtained from mice at $24 \mathrm{~h}$ post-CLP operation. In all instances, $1.5 \mathrm{ml}$ of blood was introduced in the tube not containing any anticoagulant substance. The collected blood was left on the laboratory bench for $30 \mathrm{~min}$ at room temperature prior to centrifugation $\left(3,000 \times \mathrm{g}, 5 \mathrm{~min}, 4^{\circ} \mathrm{C}\right)$, then the serum was carefully absorbed and transferred to a clean tube, and then stored at $-80^{\circ} \mathrm{C}$ as soon as possible.

ELISA. In brief, $10 \mu \mathrm{l}$ serum sample was diluted in $40 \mu \mathrm{l}$ sample dilution buffer per ELISA (Nuoyang Biotech, Hangzhou, China) plate well, and $100 \mu$ l horseradish peroxidise (HRP)-conjugated detection antibody [IL-6, tumor necrosis factor (TNF)- $\alpha$, IL-22 and IL-2] was added. The ELISA kits used were as follows: IL-6 (cat. no. CME0006-048), IL-22 (cat. no. CME0033-048) IL-2 (cat. no. CME0001-048) (Nuoyang Biotech) and TNF- $\alpha$ (cat no. ml002095; Shanghai Enzyme-linked Biotechnology Co., Ltd., Shanghai, China; http://www.mlbio.cn/).

The plate was incubated for $1 \mathrm{~h}$ at $37^{\circ} \mathrm{C}$ and washed five times. HRP-substrate goat anti-mouse IgG (cat. no. 31430; 1:100; Invitrogen; Thermo Fisher Scientific, Inc., Waltham, MA, USA) was then added and incubated for $15 \mathrm{~min}$ at $37^{\circ} \mathrm{C}$ in the dark. The enzyme reaction was then stopped with $50 \mu \mathrm{l}$ stop solution and absorbance was measured at $450 \mathrm{~nm}$. Concentrations of inflammatory factors were calculated using a standard curve generated by standard solution in different concentration. Serum levels of inflammatory factors are presented as $\mathrm{pg} / \mathrm{ml}$.

Histological analysis. Colon tissues were collected from mice at $24 \mathrm{~h}$ post-CLP operation and fixed in $10 \%$ formalin for $24 \mathrm{~h}$. The fixed colon tissues were embedded in paraffin and sectioned at $4 \mu \mathrm{m}$ thickness. The sections were stained with hematoxylin-eosin (H\&E) for histological observation to examine the pathology of colon injury induced by CLP. Immunohistochemistry staining for Ki67 and occludin were performed for measuring proliferation and tight junction formation between intestinal epithelial cells. For immunohistochemistry staining, the standard protocol was performed. Briefly, sections were incubated with $3 \% \mathrm{H}_{2} \mathrm{O}_{2} 5 \mathrm{~min}$ at room temperature to eliminate the endogenous peroxidase activity, and then blocked with $5 \%$ bovine serum albumin for $1 \mathrm{~h}$ at $37^{\circ} \mathrm{C}$ and then incubated with rabbit anti-Ki67 polyclonal antibody (cat. no. PA5-19462; 1:100; Invitrogen; Thermo Fisher Scientific, Inc.) or rabbit anti-occludin polyclonal antibody (cat. no. 404700; 1:100; Invitrogen; Thermo Fisher Scientific, Inc.) at $4^{\circ} \mathrm{C}$ overnight. This was followed by washing three times with PBS for 5 min each. The sections were incubated with horseradish peroxidase-conjugated mouse-anti-rabbit IgG secondary antibody (cat. no. 31464; 1:100; Invitrogen; Thermo Fisher Scientific, Inc.) for $1 \mathrm{~h}$ at $37^{\circ} \mathrm{C}$. Colour was developed with a 3,3'-diaminobenzidine kit (Nanjing KeyGen Biotech Co., Ltd., Nanjing, China) and microscopically examined with light microscope (Motic BA210; Motic Instruments, Richmond, $\mathrm{BC}$, Canada) using the following calculation: Ki67-positive cells/100 crypts or Occludin-positive cells, in five random x100 magnified fields, five mice per group, one section per mouse. Terminal deoxynucleotidyl-transferase-mediated dUTP-biotin nick end-labeling (TUNEL) assay (Invitrogen; Thermo Fisher Scientific, Inc.) was used for evaluation of apoptosis in the CLP-treated colon, according to the manufacturer's protocol (TUNEL-positive cells/100 crypts, in five random x100 magnified fields, five mice per group, one section per mouse).

DNA extraction and polymerase chain reaction (PCR) amplification. To ensure the abundance of LGG (normal/saline, $\mathrm{n}=5$; normal/LGG, n=5), DNA was extracted from fecal samples obtained from a separate set of mice prior to CLP operation. Quantification of LGG was performed by PCR using the following primers: LactoF, 5'-AGCAGTAGGGAATCTTCC A; and LactoR, 5'-ATTYCACCGCTACACATG. The PCR reaction was performed as follows: $95^{\circ} \mathrm{C}$ for $5 \mathrm{~min}$, followed by 35 cycles 
at $94^{\circ} \mathrm{C}$ for $15 \mathrm{sec}, 53^{\circ} \mathrm{C}$ for $30 \mathrm{sec}$, and $72^{\circ} \mathrm{C}$ for $45 \mathrm{sec}$ and a final extension at $72^{\circ} \mathrm{C}$ for $10 \mathrm{~min}$. To analyze the microbial diversity in mice with sepsis, fecal samples were collected from mice at $24 \mathrm{~h}$ post-CLP operation and frozen at $-80^{\circ} \mathrm{C}$ immediately. Microbial DNA was extracted from fecal samples using a Qiagen mini kit (Qiagen GmbH, Hilden, Germany) according to the manufacturer's instructions. The V3-V4 region of the bacteria $16 \mathrm{~S}$ ribosomal RNA gene was PCR-amplified using primers 341F (5'-CCTAYGGGRBGCASCAG-3') and 806R (5'-GGACTACNNGGGTATCTAAT-3'). An eight bp unique barcode sequence was attached to each sample. A total of $50 \mu \mathrm{l}$ PCR reactions contained $0.5 \mu \mathrm{l}$ of dNTPs $(10 \mathrm{mmol} / \mathrm{l}), 0.5 \mu \mathrm{l}$ each primer ( $5 \mu \mathrm{mol} / \mathrm{l}), 0.5 \mu \mathrm{l}$ Fast-Pfu polymerase (Beijing Transgen Biotech Co., Ltd., Beijing, China), $5 \mu 1$ 10X Fast-Pfu buffer, and $10 \mathrm{ng}$ template DNA. The PCR reaction was performed as follows: $98^{\circ} \mathrm{C}$ for $30 \mathrm{sec}$, followed by 15 cycles at $98^{\circ} \mathrm{C}$ for $10 \mathrm{sec}, 65^{\circ} \mathrm{C}$ for $30 \mathrm{sec}$, and $72^{\circ} \mathrm{C}$ for $30 \mathrm{sec}$ and a final extension at $72^{\circ} \mathrm{C}$ for $5 \mathrm{~min}$. PCR products were pooled, purified using the AxyPrep DNA gel extraction kit (Axygen Biosciences, Union City, CA, USA) according to the manufacturer's protocol, and quantified using a fluorometric kit (Quant-iT PicoGreen; Invitrogen; Thermo Fisher Scientific, Inc.). Purified products were pooled in equimolar for library construction. An Illumina miseq platform (Illumina Inc., San Diego, CA, USA) was applied to construct the paired-end sequencing (2x250 bp) and sequencing libraries according to the standard protocols.

Sequence analysis. Based on barcode and PCR primer, all raw reads were screened using the Quantitative Insights Into Microbial Ecology software (QIIME, version 1.17) (14). The $250 \mathrm{bp}$ sequences were trimmed at the site where a quality score $<20$ over a 10 bp sliding window was received, and sequences were omitted according to the following criteria: 1) Containing ambiguous sequences, 2) The maximum nucleotide mismatches in primer matching was two, 3) Sequences shorter than $150 \mathrm{bp}$. UCHIME was used to identify and remove chimeras. USEARCH was applied to generate operational taxonomic units (OTUs), reads with the maximum length in each OTU were selected as representative sequences. Based on the bacterial SILVA dataset (15), representative sequences were assigned to different taxonomic levels. The richness and diversity of gut bacteria were evaluated using indexes chao1; simpson and shannon respective. Principal coordinates analysis (PCOA) was conducted on the base of weighted and unweighted uniFrac distance metrics analysis to assess the interactions among bacterial communities of different samples. Ribosomal database project classifier (http://rdp.cme. msu.edu/) was applied to annotate the sequences.

Statistical analysis. The homogeneity of variances was verified using Bartlett's test. One-way analysis of variance (with Bonferroni as a post hoc test) was performed to compare the bacteremia, inflammatory factors and histological study variables among different groups. Survival studies were conducted via the log-rank test. Two-tailed non-parametric Kruskal-Wallis test was used to compare the differences in diversity indexes and microbial taxa. The statistical analysis was conducted using GraphPad Prism 5 (GraphPad Software, Inc., La Jolla, CA, USA). $\mathrm{P}<0.05$ was considered to indicate a statistically significant difference.
Table I. Lactobacillus in the colon of mice.

\begin{tabular}{lccc}
\hline & $\begin{array}{c}\text { Normal/LGG } \\
(\mathrm{n}=5)\end{array}$ & $\begin{array}{c}\text { Normal/saline } \\
(\mathrm{n}=5)\end{array}$ & P-value \\
\hline Lactobacillus & $9.24 \pm 0.06$ & $8.16 \pm 0.11$ & $<0.001$ \\
\hline
\end{tabular}

Quantification data from PCR analysis are expressed as log qPCR copy/fecal $(\mathrm{g})$. Data are expressed as mean \pm standard deviation. LGG, Lactobacillus rhamnosus GG; PCR, polymerase chain reaction.

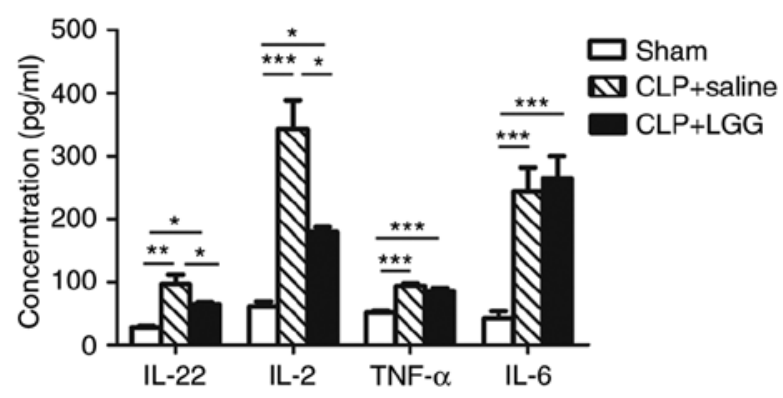

Figure 1. LGG pretreatment decreases systemic inflammatory response in sepsis. The serum levels of inflammatory factors were examined in mice with different treatment. All four inflammatory factors were the lowest in sham mice. Serum levels of IL-22 and IL-2 were decreased with LGG pretreatment, while LGG had no effect on TNF- $\alpha$ and IL-6 levels in septic mice. Data are expressed as mean \pm standard deviation $(n=10$ per group). ${ }^{*} \mathrm{P}<0.05,{ }^{* *} \mathrm{P}<0.01$ and ${ }^{* * *} \mathrm{P}<0.001$ with comparisons indicated by lines. LGG, Lactobacillus rhamnosus GG; IL, interleukin; TNF, tumor necrosis factor; CLP, cecal ligation and puncture.

\section{Results}

$L G G$ pretreatment reduces mortality and systemic inflammatory response in septic peritonitis. DNA isolated from the colon was analyzed by PCR to quantify the abundance of LGG. There was a significant increase in LGG in normal/LGG mice $(9.24 \pm 0.06, \log$ qPCR copy/fecal $(\mathrm{g}) ; \mathrm{n}=5)$ compared with normal/saline mice $(8.16 \pm 0.11, \log$ qPCR copy/fecal (g); n=5; Table I). These data indicated that LGG was able to survive and propagate in the gastrointestinal tract following oral administration for 4 weeks.

A previous study demonstrated that septic mice pretreated with LGG had a markedly improved 7 day survival rate compared with the CLP+saline mice $(\mathrm{P}=0.029)$. Five sham mice survived (10).

To evaluate the effect of LGG on reducing the inflammatory response caused by sepsis, the levels of proinflammatory cytokines IL- 6 , TNF- $\alpha$, IL-2, and of the anti-inflammatory cytokine IL-22, were detected by ELISA assay in the serum of the mice. Serum levels of IL-22, IL-2, TNF- $\alpha$ and IL-6 were the lowest in sham mice (Fig. 1). IL-22 and IL-2 levels were the highest in CLP+saline mice (Fig. 1). In the LGG-pretreated mice, IL-22 and IL-2 levels remained elevated in the septic mice compared with the sham mice $(\mathrm{P}<0.05$; Fig. 1$)$, but were decreased compared with the CLP+saline mice $(\mathrm{P}<0.05$; Fig. 1$)$. TNF- $\alpha$ and IL-6 levels were higher in septic mice compared with sham mice $(\mathrm{P}<0.001$; Fig. 1), regardless of $\mathrm{LGG}$ pretreatment or not. 

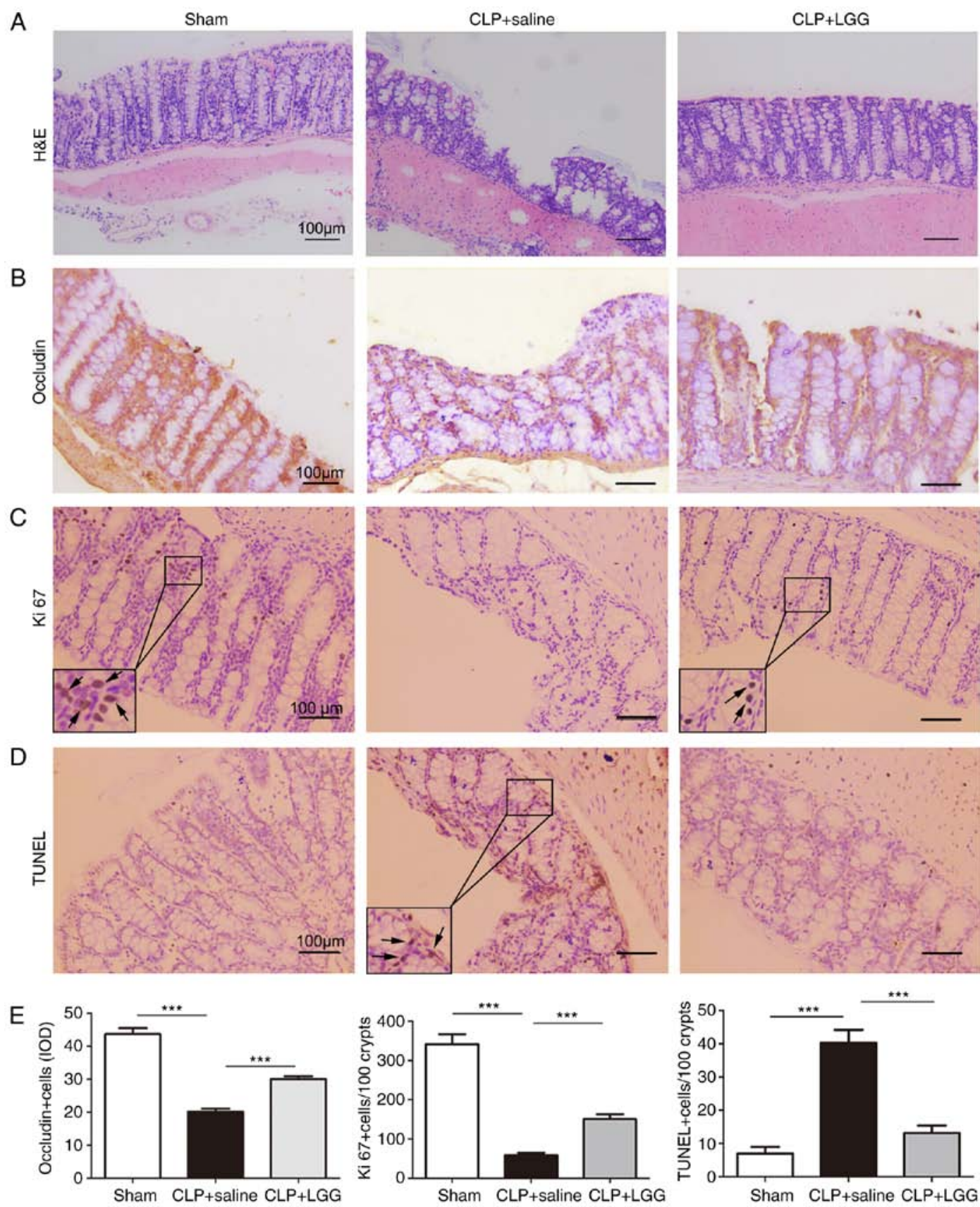

Figure 2. LGG pretreatment attenuates the injury of colon mucosa. Representative images (magnification, x100) from (A) H\&E. (B) Occludin (brown signal). (C) Ki67 (positive cells indicated by black arrows). (D) TUNEL (positive cells indicated by black arrows) staining of ileum sections from the sham, CLP+saline and CLP+LGG mouse groups and (E) Quantification of the immunohistochemistry results. Data are expressed as mean \pm standard deviation ( $\mathrm{n}=10 \mathrm{per}$ group). ${ }^{* * * *} \mathrm{P}<0.001$ with comparisons indicated by lines. LGG, Lactobacillus rhamnosus GG; CLP, cecal ligation and puncture; IOD, integrated optical density.

$L G G$ pretreatment alleviates injury in colon mucosa. As evaluated by $\mathrm{H} \& \mathrm{E}$ staining and histopathological analysis, colon tissues in sham mice were histologically normal in all layers (Fig. 2A). In CLP+saline mice, the epithelial structure was almost entirely corrupted and disintegrated, accompanied with gland deformation and infiltration of inflammatory cells (Fig. 2A). However, with LGG pretreatment, the epithelial appearance was regular, with less inflammatory cell infiltration in septic mice (Fig. 2A). Occludin staining revealed a significant decrease in tight junction formation in the colonic tissue of both CLP+saline and CLP+LGG mice, compared with the sham group (Fig. 2B). However, the level of Occludin was much higher in CLP+LGG mice compared with the
CLP+saline mice ( $\mathrm{P}<0.001$; Fig. 2B and E). Colon mucosal injury was obvious in septic mice. The damage in septic mice pretreated with LGG was less obvious compared with the CLP+saline mice. Significant differences existed in mucosal morphology and tight junction formation among CLP+LGG mice, CLP+saline mice and sham mice.

$L G G$ pretreatment normalizes cell proliferation and apoptosis in colon. In the present study, colon mucosal injury was obvious in septic mice, but the injury was alleviated by LGG pretreatment. Therefore, cell proliferation marker and apoptosis of the colonic epithelium were examined next, by Ki67 staining (Fig. 2C) and TUNEL assay (Fig. 2D), 
Table II. Summary of alpha analysis data.

\begin{tabular}{lccc}
\hline & Sham & CLP+saline & CLP+LGG \\
\hline OTUs & $1,299.60 \pm 166.56$ & $1,058.75 \pm 199.53$ & $1,184.63 \pm 343.76$ \\
Coverage & $99 \%$ & $99 \%$ & $99 \%$ \\
Chao1 & $2,151.26 \pm 230.87$ & $1,800.81 \pm 325.82$ & $1,953.06 \pm 569.96$ \\
Shannon & $6.27 \pm 0.39$ & $5.29 \pm 0.72$ & $5.84 \pm 1.12$ \\
Simpson & $0.97 \pm 0.02$ & $0.92 \pm 0.06$ & $0.94 \pm 0.07$
\end{tabular}

Data are expressed as mean \pm standard deviation. CLP, cecal ligation and puncture; LGG, Lactobacillus rhamnosus GG; OTU, operational taxonomic units.

respectively. Quantification of the results revealed a $\sim 6$-fold decrease of Ki67-positive cells $(\mathrm{P}<0.001)$ and a significant increase of TUNEL-positive cells $(\mathrm{P}<0.001)$ in CLP+saline mice compared with the sham group (Fig. 2E). Furthermore, the average numbers of Ki67-positive cells dramatically increased ( $\sim 3$-fold), while TUNEL-positive cells significantly decreased in CLP+LGG mice compared with CLP+saline mice $(\mathrm{P}<0.001$; Fig. $2 \mathrm{E})$. These results suggested that $\mathrm{LGG}$ pretreatment promoted the proliferation and decreased the apoptosis of colon tissue in septic mice.

$L G G$ pretreatment increases microbial diversity in mice with sepsis. The coverage index was $>97 \%$ per sample, which means the 16SrRNA sequences detected in the present study were sufficient to represent the majority of the bacteria present in the samples. The OTUs, richness index (Chao 1) and diversity index (Shannon and Simpson) were decreased in control septic mice compared with sham mice (Table II). With LGG pretreatment, all the four indexes increased in septic mice (Table II). Although no significant difference was observed in the four indexes, the richness and diversity of bacteria in the CLP+LGG mice was much closer to that of sham mice compared with CLP+saline mice (Table II). The result indicated that sepsis decreased microbial richness and diversity, and the trend could be reversed by LGG treatment.

Principal coordinates analysis among three groups. The weighted unifrac PCoA plot was used to compare the similarities of microbiota structure among three groups. The PCoA plot revealed a significantly separate clustering in microbiota structure among the three groups. The distance between CLP+LGG and sham mice was closer compared with the distance between CLP+saline and sham mice (PC1 and PC2 were 0.242 and 0.107 , respectively; Fig. 3).

Bacterial taxonomic differences between septic mice with different treatment. The microbial profiles of the experimental mouse groups represented 10 phyla, among which, Bacteroide, Firmicutes and Verrucomicrobia were the dominant phyla in sham mice, while Bacteroide, Proteobactria and Firmicutes composed the majority of phyla in both control CLP+saline and CLP+LGG mice (Fig. 4A and B). Compared with sham mice, the level of Firmicutes was lower, Proteobactria $(\mathrm{P}<0.01)$

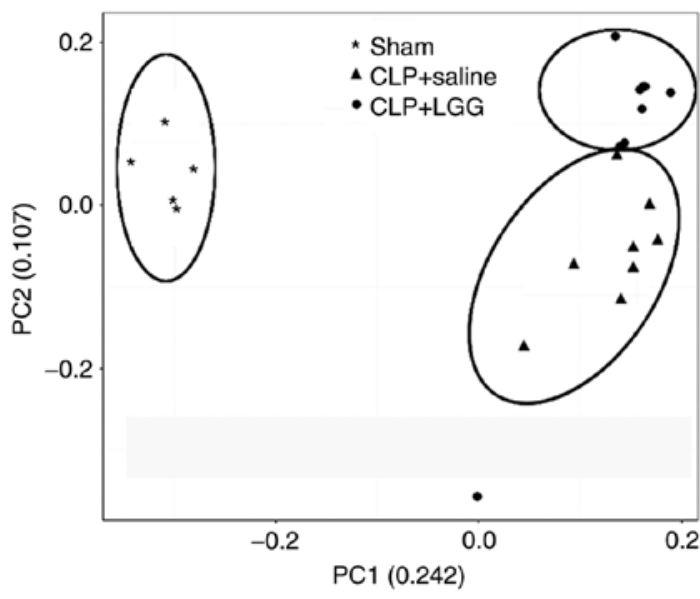

Figure 3. Principal coordinates analysis plots based on weighted unifrac metrics in mice with different treatment. Stars represent the sham group $(\mathrm{n}=5)$, triangles represent the control septic CLP+saline group $(\mathrm{n}=8)$, and dots represent the CLP+LGG group $(\mathrm{n}=8)$. LGG, Lactobacillus rhamnosus $\mathrm{GG}$; CLP, cecal ligation and puncture.

and Deferribacteres $(\mathrm{P}<0.05)$ were higher, and the ratio of Bacteroidetes/Firmicutes (B/F) increased in CLP+saline mice. With LGG pretreatment, both bacteria composition and $\mathrm{B} / \mathrm{F}$ ratio in septic mice reversed to the level of sham mice ( $\mathrm{P}>0.05$; Fig. $4 \mathrm{C}$ and $\mathrm{D})$. The majority of OTUs that were detected among the three groups of mouse microbiomes were assigned to 28 bacterial families. Among these detected taxa, Staphylococcaceae and Enterococcaceae only existed in septic mice, whereas Prevotellaceae was only present in sham mice. Verrucomicrobiaceae $(\mathrm{P}<0.05)$, S24-7 $(\mathrm{P}<0.05)$, F16 $(\mathrm{P}<0.001)$ and Alcaligenaceae $(\mathrm{P}<0.001)$ were remarkably lower and Enterobacteriaceae $(\mathrm{P}<0.01)$, Bacteroidaceae $(\mathrm{P}<0.01)$, Erysipelotrichaceae, Deferribacteraceae $(\mathrm{P}<0.05)$, Clostridiaceae $(\mathrm{P}<0.05)$ and Pseudomonadaceae $(\mathrm{P}<0.01)$ were higherinCLP+saline mice compared with shammice. WithLGG treatment, Staphylococcaceae disappeared, Enterococcaceae decreased and the other bacteria reversed to the levels of shams ( $\mathrm{P}>0.05$; Fig. 4E and $\mathrm{F})$. At genus level, Akkermansia $(\mathrm{P}>0.05)$, Coprococcus $(\mathrm{P}<0.01)$, Sutterella $(\mathrm{P}<0.001)$, Oscillospira $(\mathrm{P}>0.05)$, Lactobacillus $(\mathrm{P}<0.05)$ and Desulfovibrio $(\mathrm{P}<0.01)$ decreased, and Bacteroides $(\mathrm{P}<0.01)$, Pseudomonas $(\mathrm{P}<0.01)$, Parabacteroides, Ruminococcus $(\mathrm{P}<0.05)$, Mucispirillum $(\mathrm{P}<0.05)$ and Vagococcus $(\mathrm{P}<0.05)$ increased in CLP+saline mice compared with sham mice. With LGG pretreatment, no significant difference was observed between septic and sham mice in most of the bacteria (Fig. 4G and $\mathrm{H}$ ).

Linear discriminant analysis (LDA) effect size (LEfSe) reveals microbiota structure of the three groups and their predominant bacteria. LEfSe was used to compare the microbiota phylotypes among the three groups. The sham microbiome had a huge preponderance of Verrucomicrobia, Akkermansia, S24-7, F16 and TM_7, whereas the CLP+saline microbiome had a preponderance of Bacteroide, Enterobacteriales, Enterococcaceae, Deferribacteres, Pseudomonales and Erysipelotrichi (Fig. 5). Lactobacillales, Pseudomonales and Erysipelotrichi were enriched in CLP+LGG mice (Fig. 6). 

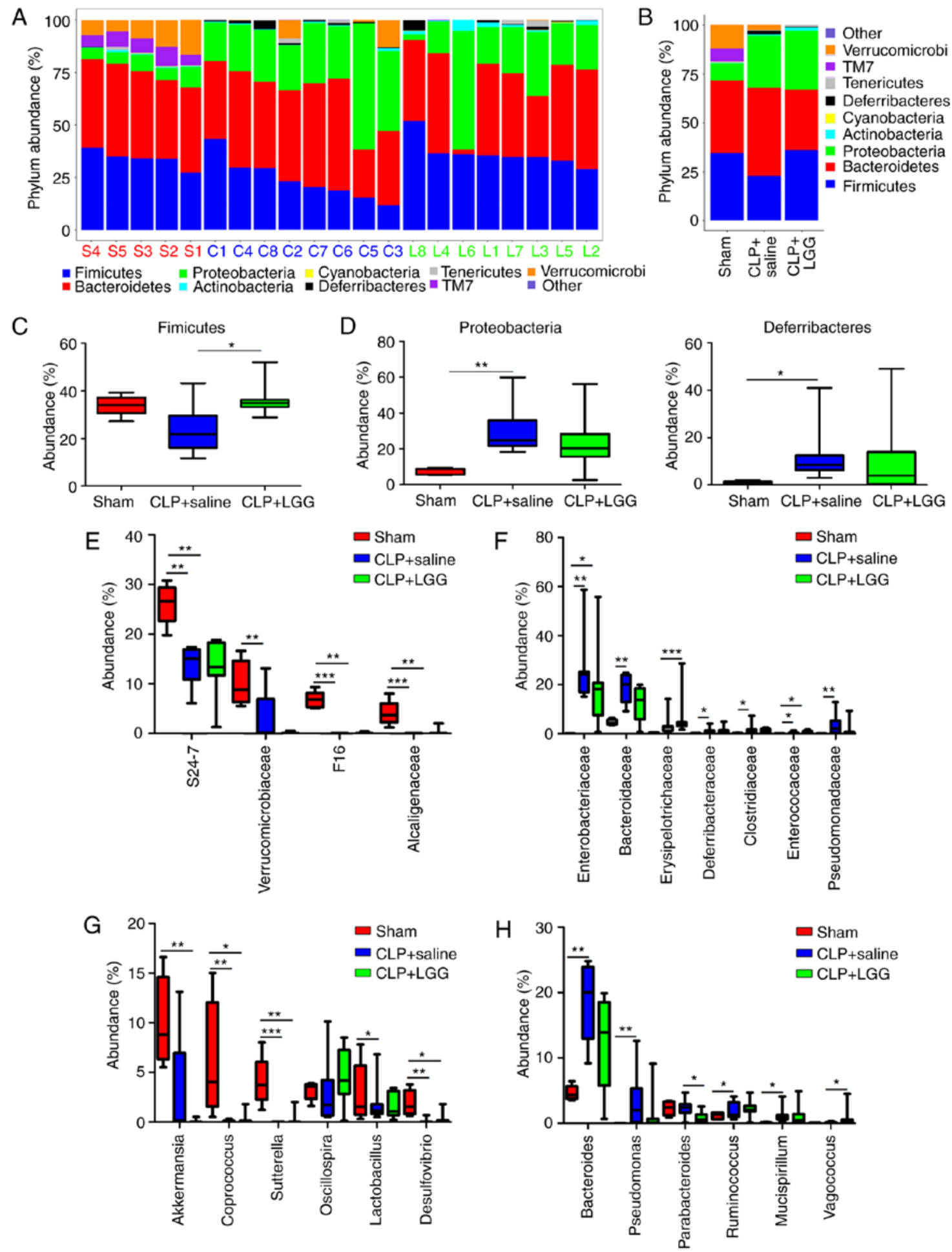

Figure 4. Comparison of the major microbiomes at the (A-D) phyla, (E and F) family and ( $\mathrm{G}$ and $\mathrm{H}$ ) genus levels between sham mice (red), CLP+saline (blue) and CLP+LGG mice (green) respectively. (A) Comparison of the abundances of bacterial phyla of each sample. (B) Comparison of the average abundance of each bacterial phylum in the sham, CLP+saline and CLP+LGG mice, respectively. (C and D) Significant differences among the abundances of discriminatory phyla among three groups. (E and G) Enrichment in sham mice; (F and H) Enrichment in CLP+saline mice; the upper and lower ranges of the box represent the 75 and $25 \%$ quartiles, respectively. Sham mice, $\mathrm{n}=5$; CLP+saline mice, $\mathrm{n}=8$; CLP+LGG mice, $\mathrm{n}=8 .{ }^{*} \mathrm{P}<0.05,{ }^{* *} \mathrm{P}<0.01$ and ${ }^{* * *} \mathrm{P}<0.001$ with comparisons indicated by lines. LGG, Lactobacillus rhamnosus GG; CLP, cecal ligation and puncture.

Compared with control septic mice, Deferribacteres disappeared, and Verrucomicrobia and Akkermansia appeared in septic mice treated with LGG (Fig. 7). These results suggested that sepsis-induced microbiota dysbiosis can be reversed by LGG pretreatment.

\section{Discussion}

Critical illness and its treatment create a hostile environment in the gastrointestinal tract by altering the microbiota. Altered mucosal oxygen gradient (16) and increased nitrate 


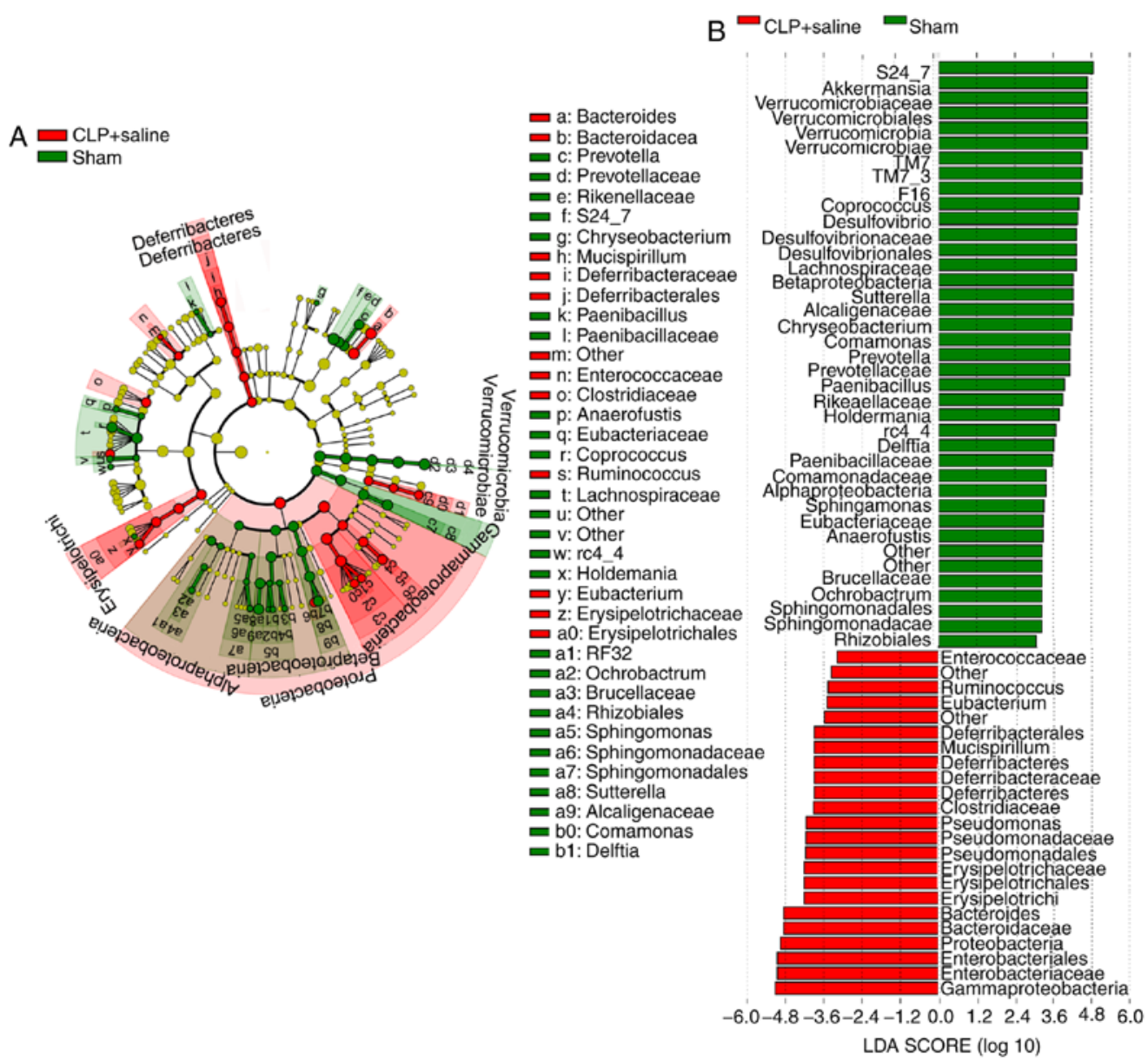

Figure 5. LEfSe and LDA analysis based on OTUs characterize the microbiomes between the sham and CLP+saline mice. (A) Cladogram using LEfSe method indicating the phylogenetic distribution of fecal microbes associated with sham (green) and CLP+saline mice (red). (B) LDA scores showed the significant bacterial difference between the sham and CLP+saline mice. Sham mice, $n=5 ; C L P+s a l i n e ~ m i c e, ~ n=8$. LEfSe, linear discriminant analysis effect size; LDA, linear discriminant analysis; OUT, operational taxonomic unit; CLP, cecal ligation and puncture.

production (17) caused by critical illness favor the growth and invasion of opportunistic pathogens, such as Pseudomonas and Escherichia coli in Proteobacteria phylum, and Staphylococcus and Enterococcus in Firmicutes phylum (18-20), resulting in the release of cytokines, cell apoptosis and corruption of epithelial tight junctions (21). With loss of intestinal barrier function, the gut is unable to prevent the translocation of pathogens and toxins into the blood and extraintestinal organs, leading to or aggravating sepsis and mortality (22). The present study demonstrated that, after the onset of sepsis, there was an appearance of opportunistic pathogens Staphylococcaceae and Enterococcaceae, and a disappearance of beneficial Prevotellaceae. High relative abundance of potentially pathogenic commensals, such as Enterobacteriaceae, Bacteroidaceae, Erysipelotrichaceae, Deferribacteraceae, Clostridiaceae and Pseudomonadaceae, was associated with more severe immune responses during sepsis, demonstrated by higher serum levels of proinflammatory cytokines (IL-22, IL-2, TNF- $\alpha$ and IL-6), epithelial cell apoptosis and disruption of tight junctions. With LGG pretreatment, opportunistic pathogens decreased or even disappeared, while beneficial bacteria, such as Verrucomicrobiaceae, increased, epithelial cell apoptosis was inhibited, and proliferation and cell tight junction formation was promoted. The present results suggest that prophylactic LGG therapy could be effective in reducing mortality from sepsis via the normalization of altered gut flora, inhibiting systemic inflammation and maintaining the mucosal barrier function.

Bacteroidetes and Firmicutes are the two dominant phylum in the human and mouse microbiome. Changes in the relative abundance of Bacteroidetes and Firmicutes have been determined to affect energy balance. Firmicutes was related with energy harvest and storage, while Bacteroidetes has a capacity to consume energy (23). The present results revealed a higher $\mathrm{B} / \mathrm{F}$ ratio in $\mathrm{CLP}+$ saline mice compared with sham mice. With LGG treatment, the B/F ratio decreased. One potential explanation for this may be that the high degree of systemic inflammation caused by sepsis is a high energy consuming process, and therefore, during this process, both energy harvest and storage decreased. As a result, Firmicutes decreased and Bacteroidetes increased. Prophylactic LGG therapy partly reversed the $\mathrm{B} / \mathrm{F}$ ratio and therefore rebalanced the energy intake and expenditure, and reduced mortality in sepsis.

Vollaard et al (24) reported the concept of colonization resistance (CR). In this concept, the anaerobic microbiome limits the concentration of opportunistic pathogenic (mostly aerobic) bacteria in the gastrointestinal tract (24). Analysis of fecal microbiome revealed that total anaerobic bacteria counts decreased notably in patients with severe systemic inflammatory 

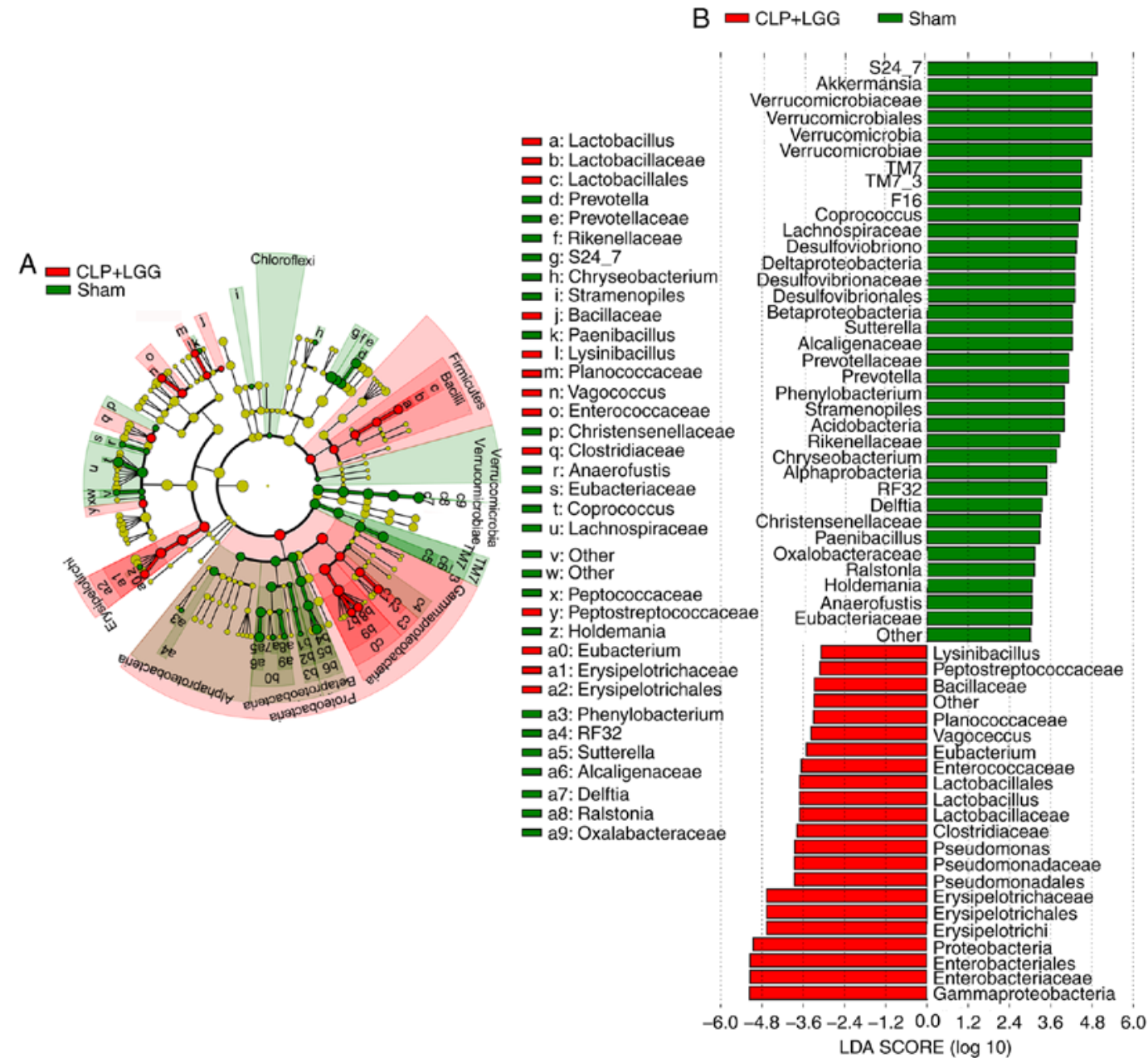

Figure 6. LEfSe and LDA analysis based on OTUs characterize the microbiomes between the sham and CLP+LGG mice. (A) Cladogram using LEfSe method indicating the phylogenetic distribution of fecal microbes associated with sham (green) and CLP+LGG mice (red). (B) LDA scores showed the significant bacterial difference between the sham and CLP+LGG mice. Sham mice, $n=5$; CLP+LGG mice, $n=8$. LEfSe, linear discriminant analysis effect size; LDA, linear discriminant analysis; OUT, operational taxonomic unit; CLP, cecal ligation and puncture; LGG, Lactobacillus rhamnosus GG.

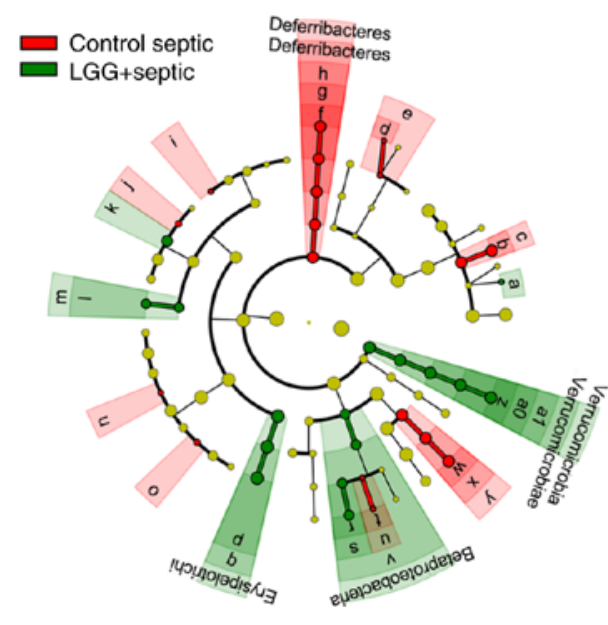

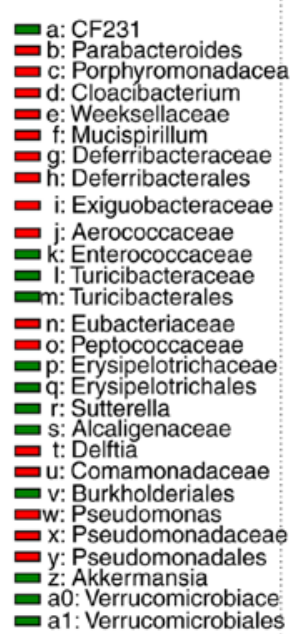

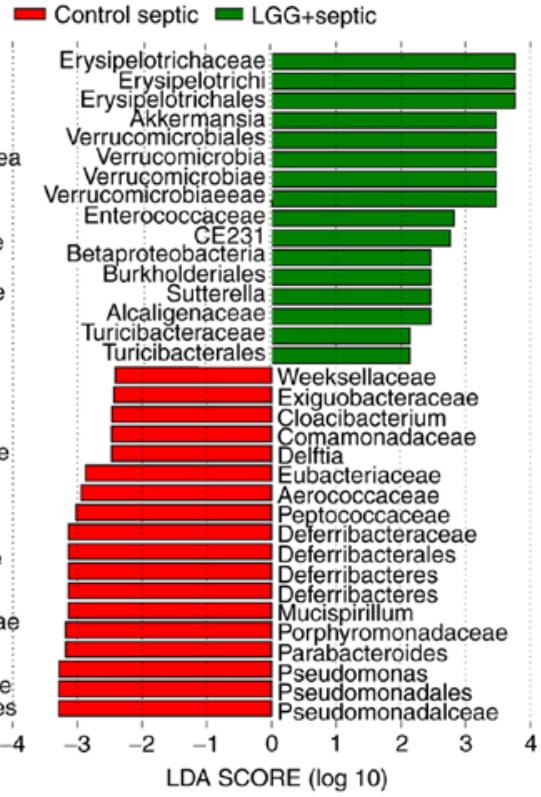

Figure 7. LEfSe and LDA analysis based on OTUs characterize the microbiomes between the CLP+saline mice and CLP+LGG mice. Cladogram using LEfSe method indicating the phylogenetic distribution of fecal microbes associated with CLP+LGG (green) and CLP+saline mice (red). LDA scores showed the significant bacterial difference between the CLP+LGG and CLP+saline mice. CLP+saline mice, $\mathrm{n}=8 ; \mathrm{CLP}+\mathrm{LGG}$ mice, $\mathrm{n}=8$. LEfSe, linear discriminant analysis effect size; LDA, linear discriminant analysis; OUT, operational taxonomic unit; CLP, cecal ligation and puncture; LGG, Lactobacillus rhamnosus GG. 
response syndrome (25). Research linking gut translocation of bacteria to the development of postoperative sepsis indicated that Gram-negative facultative anaerobic bacteria were the dominant microflora in patients with postoperative sepsis, whereas obligate anaerobes counts decreased, suggesting that the imbalance between obligate anaerobes and facultative anaerobes is closely related to the incidence of infectious complications in critically ill patients (26). In the present study, facultative anaerobic bacteria, including Enterobacteriaceae, Bacteroidaceae and Erysipelotrichaceae, increased significantly and obligate anaerobes, such as Prevotellaceae, disappeared in control septic mice. Prophylactic LGG therapy rebalanced the ratio of obligate anaerobes and facultative anaerobes, and therefore enhanced the ability of colonization resistance in the host.

Evidence suggests that several specific members of microbiota serve important roles in rebalancing dysbiosis and preventing disease $(27,28)$. For example, Coprococcus produces butyrate to provide an energy source for epithelial cells (29) and induces the differentiation of colonic regulatory $\mathrm{T}$ cells to suppress inflammatory and allergic responses (30). Akkermansia has been described to have an anti-inflammatory property. It has been reported that Akkermansia administration could raise the levels of endocannabinoids in intestinal cells that control the gut peptide secretion, gut barrier, and inflammation (31). In addition, Akkermansia, Coprococcus, Lactobacillus, Oscillibacter have been correlated negatively with lipopolysaccharide (LSP) in feces and hepatic function features (including plasma glucose and total lipids), whereas the LPS-producer Bacteroides were positively correlated with fecal LPS and hepatic function in mice with liver diseases (32). In the present study, Akkermansia, Coprococcus, Sutterella, Oscillospira, Lactobacillus and Desulfovibrio were decreased and Bacteroides were increased in control septic mice compared with sham mice at the genus level. With LGG treatment, no significant difference was observed between septic and sham mice, indicating that LGG may increase the gut barrier function, decrease the levels of LPS, reduce inflammation and improve hepatic function through increasing Akkermansia, Coprococcus, Lactobacillus, Oscillibacter and decreasing Bacteroides.

In summary, the present study demonstrated that prophylactic LGG therapy reduced mortality through attenuating inflammatory responses, and increasing gut barrier integrity and function. LGG pretreatment increased the diversity of intestinal microbiota and expression of beneficial bacterium. Among them, Prevotellaceae, Lactobacillaceae, Staphylococcaceae, Enterococcaceae, Enterobacteriaceae, Bacteroidaceae, Deferribacteraceae can be regarded as the key bacteria in sepsis treatment. Prevotellaceae may be exploited as a potential probiotic in sepsis treatment.

\section{Acknowledgements}

Not applicable.

\section{Funding}

The present study was supported by the Natural Science Foundation of China (grant no. 81771498) and by Diagnosis and treatment of gastrointestinal dysfunction in elderly patients with integrated traditional Chinese and Western Medicine (2017-XK-A31).

\section{Availability of data and materials}

The analyzed datasets generated during the study are available from the corresponding author on reasonable request. The data that support the findings of survival studies are available from reference 10 .

\section{Authors' contributions}

LC performed the histological examination of the colon, 16SrRNA analysis and was a major contributor in writing the manuscript. HL and JL performed the LGG administration and CLP model establishment. YC performed the ELISA analysis. YY was the corresponding author of this manuscript and took part in revising the manuscript. All authors read and approved the final manuscript.

\section{Ethics approval and consent to participate}

All procedures for animal care and use were approved by the Animal Care Ethics Committee of the First Affiliated Hospital, Zhejiang University (Hangzhou, China).

\section{Patient consent for publication}

Not applicable.

\section{Competing interests}

The authors declare that they have no competing interests.

\section{References}

1. Martin GS: Sepsis, severe sepsis and septic shock: Changes in incidence, pathogens and outcomes. Expert Rev Anti Infect Ther 10: 701-706, 2012,

2. Dickson RP: The microbiome and critical illness. Lancet Respir Med 4: 59-72, 2016.

3. Klingensmith $\mathrm{NJ}$ and Coopersmith CM: The gut as the motor of multiple organ dysfunction in critical illness. Crit Care Clin 32: 203-312, 2016.

4. Sánchez B,Delgado S, Blanco-Míguez A,LourençoA, Gueimonde M and Margolles A: Probiotics, gut microbiota, and their influence on host health and disease. Mol Nutr Food Res 61, 2017.

5. Krumbeck JA, Maldonado-Gomez MX, Ramer-Tait AE and Hutkins RW: Prebiotics and synbiotics: Dietary strategies for improving gut health. Curr Opin Gastroenterol 32: 110-119, 2016.

6. Arboleya S, Watkins C, Stanton C and Ross RP: Gut bifidobacteria populations in human health and aging. Front Microbiol 7: 1204, 2016.

7. Sakai F, Hosoya T, Ono-Ohmachi A, Ukibe K, Ogawa A Moriya T, Kadooka Y, Shiozaki T, Nakagawa H, Nakayama Y and Miyazaki T: Lactobacillus gasseri SBT2055 induces TGF- $\beta$ expression in dendritic cells and activates TLR2 signal to produce IgA in the small intestine. PLoS One 9: e105370, 2014.

8. Jo SG, Noh EJ, Lee JY, Kim G, Choi JH, Lee ME, Song JH, Chang JY and Park JH: Lactobacillus curvatus WiKim38 isolated from kimchi induces IL-10 production in dendritic cells and alleviates DSS-induced colitis in mice. J Microbiol 54: 503-509, 2016.

9. Besselink MG, van Santvoort HC, Buskens E, Boermeester MA, van Goor H, Timmerman HM, Nieuwenhuijs VB, Bollen TL, van Ramshorst B, Witteman BJ, et al: Probiotic prophylaxis in predicted severe acute pancreatitis: A randomised, double-blind, placebo-controlled trial. Lancet 371: 651-659, 2008. 
10. Chen L, Xu K, Gui Q, Chen Y, Chen D and Yang Y: Probiotic pre-administration reduces mortality in a mouse model of cecal ligation and puncture-induced sepsis. Exp Ther Med 12: $1836-1842,2016$

11. von Martels JZ, Sadaghian Sadabad M, Bourgonje AR, Blokzijl T, Dijkstra G, Faber KN and Harmsen HJM: The role of gut microbiota in health and disease: In vitro modeling of host-microbe interactions at the aerobe-anaerobe interphase of the human gut. Anaerobe 44: 3-12, 2017.

12. Das S, Dash HR, Mangwani N, Chakraborty J and Kumari S: Understanding molecular identification and polyphasic taxonomic approaches for genetic relatedness and phylogenetic relationships of microorganisms. J Microbiol Methods 103 : 80-100, 2014.

13. Rittirsch D, Huber-Lang MS, Flierl MA and Ward PA: Immunodesign of experimental sepsis by cecal ligation and puncture. Nat Protoc 4: 31-36, 2009.

14. Caporaso JG, Kuczynski J, Stombaugh J, Bittinger K, Bushman FD, Costello EK, Fierer N, Peña AG, Goodrich JK, Gordon JI, et al: QIIME allows analysis of high-throughput community sequencing data. Nat Methods 7: 335-336, 2010.

15. Glöckner FO, Yilmaz P, Quast C, Gerken J, Beccati A, Ciuprina A, Bruns G, Yarza P, Peplies J, Westram R and Ludwig W: 25 years of serving the community with ribosomal RNA gene reference databases and tools. J Biotechnol 261: 169-176, 2017.

16. Albenberg L, Esipova TV, Judge CP, Bittinger K, Chen J, Laughlin A, Grunberg S, Baldassano RN, Lewis JD, Li H, et al: Correlation between intraluminal oxygen gradient and radial partitioning of intestinal microbiota. Gastroenterology 147: 1055-1063, 2014.

17. Winter SE, Winter MG, Xavier MN, Thiennimitr P, Poon V, Keestra AM, Laughlin RC, Gomez G, Wu J, Lawhon SD, et al: Host-derived nitrate boosts growth of E. coli in the inflamed gut. Science 339: 708-711, 2013

18. Lupp C, Robertson ML, Wickham ME, Sekirov I, Champion OL, Gaynor EC and Finlay BB: Host-mediated inflammation disrupts the intestinal microbiota and promotes the overgrowth of Enterobacteriaceae. Cell Host Microbe 2: 119-129, 2007.

19. Honda K and Littman DR: The microbiome in infectious disease and inflammation. Annu Rev Immunol 30: 759-795, 2012.

20. Grootjans J, Lenaerts K, Derikx JP, Matthijsen RA, de Bruïne AP, van Bijnen AA, van Dam RM, Dejong CH and Buurman WA: Human intestinal ischemia-reperfusion-induced inflammation characterized: Experiences from a new translational model. Am J Pathol 176: 2283-2291, 2010.
21. Alverdy JC, Laughlin RS and Wu L: Influence of the critically ill state on host-pathogen interactions within the intestine: Gut-derived sepsis redefined. Crit Care Med 31: 598-607, 2003.

22. Mittal R and Coopersmith CM: Redefining the gut as the motor of critical illness. Trends Mol Med 20: 214-223, 2014.

23. Turnbaugh PJ,Ley RE, Mahowald MA, Magrini V, Mardis ER and Gordon JI: An obesity-associated gut microbiome with increased capacity for energy harvest. Nature 444: 1027-1031, 2006.

24. Vollaard EJ and Clasener HA: Colonization resistance. Antimicrob Agents Chemother 38: 409-414, 1994.

25. Shimizu K, Ogura H, Goto M, Asahara T, Nomoto K, Morotomi M, Yoshiya K, Matsushima A, Sumi Y, Kuwagata Y, et al: Altered gut flora and environment in patients with severe SIRS. J Trauma 60: 126-133, 2006.

26. O'Boyle CJ, MacFie J, Mitchell CJ, Johnstone D, Sagar PM and Sedman PC: Microbiology of bacterial translocation in humans. Gut 42: 29-35, 1998.

27. Lynch SV and Pedersen O: The human intestinal microbiome in health and disease. N Engl J Med 375: 2369-2379, 2016.

28. Shreiner AB, Kao JY and Young VB: The gut microbiome in health and in disease. Curr Opin Gastroenterol 31: 69-75, 2015.

29. Donohoe DR, Garge N, Zhang X, Sun W, O'Connell TM, Bunger MK and Bultman SJ: The microbiome and butyrate regulate energy metabolism and autophagy in the mammalian colon. Cell Metab 13: 517-526, 2011

30. Furusawa Y,Obata Y,Fukuda S,Endo TA, Nakato G, Takahashi D, Nakanishi Y, Uetake C, Kato K, Kato T, et al: Commensal microbe-derived butyrate induces the differentiation of colonic regulatory T cells. Nature 504: 446-450, 2013.

31. Everard A, Belzer C, Geurts L, Ouwerkerk JP, Druart C, Bindels LB, Guiot Y,Derrien M, Muccioli GG, Delzenne NM, et al: Cross-talk between Akkermansia muciniphila and intestinal epithelium controls diet-induced obesity. Proc Natl Acad Sci USA 110: 9066-9071, 2013.

32. Xie G, Wang X, Liu P, Wei R, Chen W, Rajani C, Hernandez BY, Alegado R, Dong B, Li D and Jia W: Distinctly altered gutmicrobiota in the progression of liver disease. Oncotarget 7: 19355-19366, 2016.

This work is licensed under a Creative Commons Attribution-NonCommercial-NoDerivatives 4.0 International (CC BY-NC-ND 4.0) License. 